\title{
Perfil microbiológico subgingival de pacientes con periodontitis crónica en una población de Colombia ${ }^{1}$
}

\section{Subgingival microbiological profile of patients with chronic periodontitis in a population of Colombia}

\author{
ARDILA MEDINA CM* \\ ARBELÁEZ MONTOYA MI** \\ GUZMÁN ZULUAGA IC***
}

\begin{abstract}
Ardila Medina CM, Arbeláez Montoya MI, Guzmán Zuluaga IC. Perfil microbiológico subgingival de pacientes con periodontitis crónica en una población de Colombia. Av Periodon Implantol. 2012;24, $1: 47-53$.
\end{abstract}

\section{RESUMEN}

Introducción: es necesario un entendimiento global de la composición bacteriana de la infección periodontal que permita el establecimiento de estrategias preventivas y terapéuticas particulares. Objetivo: investigar la composición de la microflora subgingival de pacientes con periodontitis crónica en una población colombiana.

Materiales y métodos: se examinaron los parámetros clínicos y la presencia de microorganismos subgingivales en 76 pacientes con periodontitis crónica en Medellín, Colombia. Las muestras subgingivales se procesaron mediante cultivo. Se utilizaron pruebas de chi cuadrado y Mann-Whitney para determinar las diferencias entre las variables clínicas con las sociodemográficas $(p<0,05)$.

Resultados y conclusiones: Se encontró mayor pérdida de inserción en los hombres y también porcentajes más elevados de placa, supuración, movilidad, y compromiso de furcación comparado con las mujeres. Los microorganismos más prevalentes fueron $P$. gingivalis $(64,4 \%)$, F nucleatum (46.3\%) y P. intermedia/nigrescens (44,3\%). Se observaron bacilos entéricos gram-negativos en 20 $(26,31 \%)$ de 76 pacientes. La prevalencia de levaduras en las bolsas periodontales de pacientes con periodontitis crónica fue de 13,2\% (10/76). Estos hallazgos tienen implicaciones terapéuticas importantes que deben tenerse en cuenta para el manejo de los pacientes periodontales en Colombia.

PALABRAS CLAVE: Periodontitis crónica, placa subgingival, periodontopatógenos, entéricos, levaduras.

\section{SUMMMARY}

Introduction: a global understanding of the bacterial composition of periodontal infection is necessary to allow the establishment of specific preventive and therapeutic strategies.

Objective: to investigate the composition of the subgingival microflora of patients with chronic periodontitis in a Colombian population

Materials and methods: parameters were examined and the presence of subgingival microorganisms in 76 patients with chronic periodontitis in Medellin, Colombia. Subgingival samples were processed by culture. We used chi square test and Mann-Whitney test to determine differences between the clinical variables with sociodemographic $(p<0.05)$.

Results and conclusions: We found a higher insertion loss in men and higher rates of plaque, suppuration, mobility, and furcation commitment compared with women. The most prevalent

Investigación financiada por la Facultad Nacional de Salud Pública y el Grupo de Epidemiología de la Universidad de Antioquía.

* Profesor Asociado Facultad de Odontología Universidad de Antioquía. Candidato a Ph. D en Epidemiología Universidad de Antioquía. Grupo de Epidemiología. Universidad de Antioquía.

** Bacterióloga de la Universidad de Antioquía. Profesora de la Facultad de Odontología. Universidad de Antioquía.

*** Periodoncista. Universidad de Chile. Profesora de la Facultad de Odontología. Universidad de Antioquía. 
microorganisms were $P$. gingivalis (64.4\%), F. nucleatum (46.3\%) and P. intermediate/nigrescens $(44.3 \%)$. We found Gram-negative enteric rods in $20(26.31 \%)$ of 76 patients. The prevalence of yeasts in periodontal pockets of patients with chronic periodontitis was $13.2 \%$ (10/76). These findings have important therapeutic implications that must be considered for the management of periodontal patients in Colombia.

KEY WORDS: Chronic periodontitis, subgingival plaque, periodontopathogens, enteric, yeast.

Fecha de recepción: 10 de jseptiembre 2009.

Fecha de aceptación: 14 de septiembre 2009.

\section{INTRODUCCIÓN}

Entre las enfermedades más difundidas en el mundo se encuentran las periodontales que pueden afectar al $48 \%$ de la población adulta, prevalencia que varía según condiciones culturales, sociales, económicas y políticas (1). Dentro del grupo de las enfermedades periodontales se encuentra la periodontitis crónica, patología infecciosa caracterizada por la formación de bolsas, que puede afectar un número variable de dientes. Además, ocasiona inflamación de los tejidos de soporte dental, pérdida ósea y de inserción (2). Las lesiones pueden ser tan graves que pueden ocasionar pérdida de los dientes y alteración de la función masticatoria y estética, afectando por consiguiente el bienestar y calidad de vida de las personas (3).

Se ha documentado extensamente el papel de la microflora subgingival en la etiología de la periodontitis crónica $(4,5)$. Diferentes estudios han demostrado similitudes en los periodontopatógenos y han encontrado que en los pacientes con periodontitis crónica están presentes y en forma conjunta la mayoría de los siguientes microorganismos: Porphyromona gingivalis, Aggregatibacter actinomycetemcomitans, Tannerella forsythensis, Eikenella corrodens, Campylobacter rectus, Peptoestreptococo micros, Treponema denticola y la Prevotella intermedius $(6,7)$. Sin embargo, algunas investigaciones han demostrado que la frecuencia relativa de cada microorganismo varía entre poblaciones de diferentes orígenes geográficos, concluyendo que la prevalencia de patógenos periodontales específicos cambia entre individuos del mismo ambiente y entre distintas etnias y países (8-13). Además de los ya mencionados, en los ambientes subgingivales de algunos pacientes con periodontitis crónica, se han encontrado otros microorganismos tales como los bacilos entéricos Gram-negativos y levaduras. No obstante, la frecuencia de este fenómeno también es diversa entre diferentes regiones del mundo $(9,14-19)$.
La mayor parte del conocimiento disponible sobre la composición microbiana de la placa subgingival se basa en estudios realizados en Europa y Estados Unidos $(20,21)$, de tal manera que este tipo de información es limitada en países latinoamericanos (8). Aun cuando existen diferencias en la composición de la microflora subgingival en sujetos de varias regiones geográficas, es necesario un entendimiento global de la composición bacteriana de la infección periodontal que permita el establecimiento de estrategias preventivas y terapéuticas particulares (8). El objetivo de este estudio fue investigar la composición de la microflora subgingival de pacientes con periodontitis crónica en una población colombiana.

\section{MATERIALES Y MÉTODOS}

\section{Sujetos}

Se invitaron a participar en este estudio 76 sujetos sistémicamente sanos (45 mujeres y 31 hombres) que asistieron a las clínicas odontológicas de la Facultad de Odontología de la Universidad de Antioquía entre Octubre de 2008 y Marzo de 2009. Cada participante firmó un consentimiento informado. El Comité de Ética de la Sede de Investigación Universitaria de la Universidad de Antioquía aprobó el diseño del estudio teniendo en cuenta la Declaración de Helsinki sobre experimentación que involucra seres humanos. Se consideraron candidatos para participar en la presente investigación, pacientes con diagnóstico de periodontitis crónica. Los criterios de exclusión fueron embarazo, lactancia, presencia de diabetes o cualquier enfermedad sistémica que alterara el curso de la enfermedad periodontal, terapia periodontal en el último año, y utilización de antimicrobianos o antiinflamatorios no esteroides en los seis meses previos al examen clínico y a la toma de muestras microbiológicas. 


\section{Evaluación clínica}

A cada paciente se le realizó una historia clínica, además de un examen clínico y radiográfico completo. Uno de los autores $(\mathrm{CA})$ realizó todos los exámenes clínicos. La profundidad de sondaje y el nivel de inserción clínica se midieron en seis sitios (mesiobucal, bucal, distobucal, distolingual, lingual y mesiolingual) usando una sonda periodontal calibrada (UNC-15, HuFriedy, Chicago, IL). El diagnóstico de periodontitis crónica se realizó según los criterios de la Academia Americana de Periodoncia (AAP) (22).

\section{Muestreo microbiológico}

Se tomaron muestras microbiológicas de los pacientes en sitios con una profundidad de sondaje $\geq 5 \mathrm{~mm}$. Para el muestreo se seleccionaron las seis bolsas periodontales más profundas. Después de aislar la zona con algodón y remover la placa supragingival con cureta, se insertaron conos de papel estéril en cada bolsa periodontal durante 20 segundos. Las muestras de cada paciente se depositaron en $2 \mathrm{~mL}$ de medio de transporte (Viability Medium Göteborg Anaerobically III: VMGA III) (23) y se llevaron al laboratorio de microbiología de la Facultad de Odontología de la Universidad de Antioquía para procesarlas dentro de las dos horas siguientes. Las muestras fueron analizadas utilizando técnicas de cultivo para la presencia de periodontopatógenos de acuerdo a Slots (24). Brevemente, para la identificación de Porphyromonas gingivalis, Prevotella intermedia/nigrescens, Prevotella melaninogenica, Tannerella forsythensis, Eikenella corrodens y Fusobacterium spp se realizaron cinco diluciones en base 10 a partir del medio VMGA III, se sembraron 100 $\mu \mathrm{L}$ de las diluciones $10^{-3}, 10^{-4}$ y $10^{-5}$ en agar brucella sangre enriquecido con hemina y menadiona (BBL Microbiology Systems, Cockeysville, MD) y se llevaron a incubación a $36^{\circ} \mathrm{C}$ en atmósfera de anaerobiosis (Anaerogen, Oxoid, Hampshire, England) durante siete días. Para la identificación de Aggregatibacter actinomycetemcomitans, las muestras sin diluir y $10^{-1}$ se sembraron en agar TSBV (tripticasa soya bacitracina vancomicina) y se incubaron en atmósfera de $10 \%$ de $\mathrm{CO}_{2}$ (Campygen, Oxoid, Hampshire, England) durante tres a cinco días. Se realizaron además pruebas bioquímicas y enzimáticas confirmatorias para todos los microorganismos utilizando los sistemas de identificación comercial Rapid ANA II (RemelTM, Apogent) y API ZYM (Biomerieux® Francia). Las cepas presuntivas de A. actinomycetemcomitans fueron identificadas por la presencia de una estructura semejante a una estrella en el interior de las colonias y las pruebas catalasa positiva y MUG negativa (4-Metilumbeliferil-B-Dgalactosido, para investigar la fermentación de la lactosa).

Para el aislamiento de bacilos entéricos gram-negativos se utilizó agar MacConkey para incubarse luego a una atmósfera aeróbica a $37^{\circ} \mathrm{C}$ por $24-48$ horas. Sobre las colonias que crecieron en agar MacConkey se empleó tinción de gram para verificación.

Para identificar las levaduras se subcultivó la colonia presuntiva en agar TSBV al agar sangre evaluándose el aspecto de la colonia y la morfología microscópica correspondiente a blastoconidias en la preparación con azul de lactofenol (25).

Después de la incubación se calculó el número total de unidades formadoras de colonias por mililitro de cada muestra a partir de los medios de cultivo. El porcentaje relativo de recuperación de cada microorganismo se calculó a partir del total de unidades formadoras de colonias por mililitro (UFC/ml).

\section{Análisis estadístico}

Para describir las variables relacionadas con los sujetos y los dientes se realizó un análisis exploratorio sobre la distribución de los índices profundidad de sondaje y nivel de inserción, utilizando medidas de tendencia central y de dispersión. Se obtuvieron frecuencias y proporciones de cada uno de los microorganismos estudiados. Para las variables habito de fumar, placa bacteriana, sangrado al sondaje, supuración, compromiso de furcaciones y movilidad dental se calcularon frecuencias y proporciones. Se utilizaron pruebas de chi cuadrado y Mann-Whitney para determinar las diferencias de las variables clínicas con las sociodemográficas $(\mathrm{p}<0,05)$. Se utilizó un programa estadístico para todos los análisis (SPSS, versión 15, Chicago, IL).

\section{RESULTADOS}

Se estudiaron 31 hombres (41\%) y 45 mujeres (59\%) con periodontitis crónica. La tabla 1 presenta las características clínicas de los pacientes evaluados. Los hombres y mujeres examinados mostraron parámetros clínicos diferentes. Se encontró mayor pérdida de inserción en los hombres y también porcentajes más elevados de placa, supuración, movilidad, y compromiso de furcación comparado con las mujeres (diferencias 


\section{TABLA 1.- CARACTERÍSTICASS CLÍNICAS DE 76 PACIENTES CON PERIODONTITIS CRÓNICA}

\begin{tabular}{|l|c|c|c|}
\hline Parámetro & Hombres & Mujeres & Valor p \\
\hline Edad & $47 \pm 9$ años & $45 \pm 8 \mathrm{años}$ & 0,5 \\
\hline Profundidad bolsa & $3,1 \pm 1,3 \mathrm{~mm}$ & $2,9 \pm 1,2 \mathrm{~mm}$ & 0,5 \\
\hline Nivel inserción & $3,8 \pm 1,2 \mathrm{~mm}$ & $3,3 \pm 1,6 \mathrm{~mm}$ & $<0,001$ \\
\hline Sangrado & $74 \%$ & $73 \%$ & 0,5 \\
\hline Placa & $56 \%$ & $48 \%$ & $<0,001$ \\
\hline Supuración & $17 \%$ & $13 \%$ & 0,006 \\
\hline Movilidad & $25 \%$ & $20 \%$ & 0,014 \\
\hline Compromiso de furcaciones & $19 \%$ & $16 \%$ & 0,05 \\
\hline Hábito de fumar & $29 \%$ & $18 \%$ & $<0,001$ \\
\hline$\pm:$ Desviación estándar & & & \\
\hline
\end{tabular}

estadísticamente significativas). Además se observó un mayor porcentaje de hombres fumadores $(p<0,001)$

Un total de 188 colonias fueron completamente identificadas en los 76 pacientes estudiados (Tabla 2). Los microorganismos más prevalentes fueron $P$. gingivalis
(64,4\%), F.nucleatum (46,3\%) y P. intermedia/nigrescens $(44,3 \%)$ (Tabla 3).

Se encontraron bacilos entéricos gram-negativos en $20(26,31 \%)$ de 76 pacientes. Se observaron especies Enterobacteriaceae en $16(21,05 \%)$ y Pseudomonas

\section{TABLA 2.- NÚMERO DE COLONIAS BACTERIANAS IDENTIFICADAS EN 76 PACIENTES CON PERIODONTITIS CRÓNICA}

\begin{tabular}{|l|c|}
\hline Microorganismo & Colonias \\
\hline Porphyromonas gingivalis & 51 \\
\hline Fusobacterium nucleatum & 36 \\
\hline Prevotella intermedia/nigrescens & 34 \\
\hline Bacilos entéricos gram-negativos & 20 \\
\hline Aggregatibacter actinomycetemcomitans & 18 \\
\hline Prevotella melaninogénica & 11 \\
\hline Levaduras & 10 \\
\hline Eikenella Corrodens & 5 \\
\hline Tanerella forsythia & 3 \\
\hline
\end{tabular}

\begin{tabular}{|l|c|}
\hline \multicolumn{2}{|c|}{ TABLA 3.- PREVALENCIA DE } \\
MICROORGANISIMOS EN 76 PACIENTES \\
CON PERIODONTITIS CRÓNICA \\
\hline Microorganismo & Prevalencia \\
\hline Porphyromonas gingivalis & $64,4 \%$ \\
\hline Fusobacterium nucleatum & $46,3 \%$ \\
\hline Prevotella intermedia/nigrescens & $44,2 \%$ \\
\hline Bacilos entéricos gram-negativos & $26,3 \%$ \\
\hline Aggregatibacter & \\
\hline actinomycetemcomitans & $23,7 \%$ \\
\hline Prevotella melaninogénica & $14,2 \%$ \\
\hline Levaduras & $13,2 \%$ \\
\hline Eikenella Corrodens & $6,5 \%$ \\
\hline Tanerella forsythia & $3,9 \%$ \\
\hline
\end{tabular}


aeruginosa en $4(5,26 \%)$ de 20 aislamientos. Se encontró Klebsiella pneumoniae en 12 pacientes y Serratia marcescens en dos pacientes. Se identificaron también otras dos especies de Enterobacteriaceae (Tabla 4).

La prevalencia de levaduras en las bolsas periodontales de pacientes con periodontitis crónica fue de $13,2 \%$ (10/76). Se encontraron ocho sujetos con C. albicans y dos pacientes con especies de Candida no específicas.

\section{DISCUSIÓN}

Este estudio investigó la composición microbiológica subgingival de 76 pacientes con periodontitis crónica en una población de Colombia. El microorganismo mas prevalente en este estudio fue $P$. gingivalis $(64,4 \%)$, frecuencia similar a la de un estudio realizado recientemente en Colombia (18). Otros estudios muestran también gran prevalencia de este microorganismo en pacientes con periodontitis crónica $(8,26-29)$. Diferentes investigaciones utilizando técnicas de cultivo encontraron rangos entre el $13 \%$ y el $87 \%$ (26-29), corroborando los resultados obtenidos en la presente investigación.

Los niveles de $A$. actinomycetemcomitans observados en pacientes con periodontitis crónica difieren entre las poblaciones estudiadas y su prevalencia varía entre el $8 \%$ y el $57 \%$ en Norteamericanos, Europeos y Asiáticos (30-32). Este estudio mostró una prevalencia del $18 \%$ similar a la obtenida por un estudio realizado recientemente en Colombia (18). Es importante tener en cuenta que el $A$. actinomycetemcomitans ha mostra-

\begin{tabular}{|l|c|}
\hline \multicolumn{2}{|c|}{ TABLA 4.- ESPECIES DE BACILOS } \\
ENTÉRICOS GRAIM-NEGATIVE \\
AISLADOS EN 20 (26,3\%) DE 76 \\
PACIENTES CON PERIODONTITIS \\
CRÓNICA \\
\hline Especie & Número (\%) \\
\hline Klebsiella pneumoniae & $12(60)$ \\
\hline Pseudomonas aeruginosa & $4(20)$ \\
\hline Serratia marcescens & $2(10)$ \\
\hline Klebsiella oxytoca & $1(5)$ \\
\hline Enterobacter sakazakii & $1(5)$ \\
\hline
\end{tabular}

do baja respuesta a la terapia mecánica periodontal (33). En este contexto, es importante estudiar su presencia en esta población debido a que diferentes protocolos antimicrobianos se administran como terapia adjunta al rapado y alisado radicular para el tratamiento de bolsas periodontales que contienen $A$. actinomycetemcomitans (34).

Desde hace casi dos décadas se ha descrito la asociación entre bacilos entéricos gram-negativos y periodontopatógenos (14). Además de reconocerse como microorganismos superinfectantes se han asociado con resistencia antibiótica a la amoxicilina y metronidazol, antimicrobianos usados frecuentemente en el tratamiento de la periodontitis (35). Latinoamérica es conocida por la prescripción indiscriminada de antibióticos lo cual puede favorecer un posible sobre crecimiento de estos patógenos en las bolsas periodontales (8). Los bacilos entéricos gram-negativos observados en este estudio $(26,31 \%)$ presentaron una prevalencia similar a la informada por otros investigadores en Colombia $(8,17,18)$ y Brasil $(16,36)$. Se requieren futuros estudios que clarifiquen el efecto de los entéricos sobre los parámetros clínicos y la respuesta al tratamiento periodontal.

La prevalencia de levaduras en esta investigación $(13.2 \%)$ fue similar a la encontrada por Slots y colaboradores (37) quienes registraron 13-14\% de levaduras en bolsas periodontales de pacientes con periodontitis refractaria. El papel que desempeñan las levaduras en la periodontitis crónica no es muy claro y se requieren más estudios para demostrar el significado clínico de sus hallazgos.

La presencia de determinados patógenos periodontales o la asociación de periodontopatógenos con microorganismos superinfectantes deben estipular la selección de los antibióticos (8). Por esta razón es fundamental conocer la composición de la microflora subgingival específica de cada región geográfica para determinar el protocolo terapéutico apropiado.

\section{CONCLUSIONES}

La composición microbiana subgingival en pacientes con periodontitis crónica de Colombia demuestra altos niveles de $P$. gingivalis, bacilos gram-negativos entéricos y levaduras. Los resultados del presente estudio muestran que los pacientes con periodontitis crónica estudiados en esta investigación presentan una microflora subgingival específica que puede estar li- 
gada a factores geográficos y ambientales particulares. Este hallazgo tiene implicaciones terapéuticas importantes que deben tenerse en cuenta para el manejo de los pacientes periodontales en Colombia.

\section{BIBLIOGRAFÍA}

1. Albandar JM. Epidemiology and risk factors of periodontal diseases. Dent Clin North Am 2005;49:517-32.

2. Armitage G. Development of a Classification System for Periodontal Diseases and Conditions. Ann Periodontol 1999;4:1-6.

3. Organización Mundial de la Salud. World Health Organization. Oral health surveys. Basic methods. Geneva (Switzerland):World Health Organization; 1997.

4. Socransky SS, Haffajje AD. Evidence of bacterial etiology: a historical perspective. Periodontol 2000 1994;5:134-44.

5. Van Winkelhoff, Herrera D, Oteo A, Sanz M. Antimicrobial profiles of periodontal pathogens isolated from periodontitis patients in the Netherlands and Spain. J Clin Periodontol 2005;32: 893-8.

6. Ali RW, Velcescu C, Jivanescu MC. Prevalence of 6 putative periodontal pathogens in subgingival plaque samples from Romanian adult periodontitis patients. J Clin Periodontol 1996;23:133-9.

7. Socransky SS, Haffajje AD, Cugini M.A, Smith CM, Kent $\mathrm{RL}$ Jr. Microbial complexes in subgingival plaque. J Clin Periodontol 1998;25:134-44.

8. Herrera D, Contreras A, Gamonal J, Otero A, Jaramillo A, Silva $\mathrm{N}$ et al. Subgingival microbial profiles in chronic periodontitis patients from Chile, Colombia and Spain. J Clin Periodontol 2008;35:106-13.

9. Sanz M, van Winkelhoff AJ, Herrera D, Dellemijn-Kippuw $\mathrm{N}$, Simon R, Winkel E. Differences in the composition of the subgingival microbiota of two periodontitis populations of different geographic location. A comparison between Spain and The Netherlands. Eur J Dent Sci 2000;108:383-92.

10. Sanz M, Lau L, Herrera D, Morillo J M, Silva A. Methods of detection of Actinobacillus actinomycetemcomitans, Porphyromonas gingivalis and Tannerella forsythensis in periodontal microbiology, with special emphasis on advanced molecular techniques: a review. J Clin Periodontol 2004;31:1034-47.

11. Umeda M, Chen C, Bakker I, Contreras A, Morrison JL, Slots J. Risk indicators for harboring periodontal pathogens. J Periodontol 1998;69:1111-18.

12. Haffajje $A D$, Borgen A, Hasturk H, Feres M, López NJ, Socransky SS. Subgingival microbiota of chronic periodontitis subjects from different geographic locations. J Clin Periodontol 2004;31:996-1002.

13. Lopez NJ, Socransky SS, Da SI, Japlit MR, Haffajee AD. Subgingival microbiota of chilean patients with chronic periodontitis. J Periodontol 2004;75:717-25.

14. Slots J, Feik D, Rams TE. Prevalence and antimicrobial susceptibility of Enterobacteriaceae, Pseudomonadaceae and Acinetobacter in human periodontitis. Oral Microbiol Inmunol 1990;5:149-54

15. Ali RW, Bakken V, Nilsen R, Skaug N. Comparative detection frecuency of six putative periodontal pathogens in Sudanese and Norwegian adult periodontitis patients. J Periodontol 1994;65:1046-52.

16. Barbosa FCB, Mayer MPA, Saba-Chuifi E, Cai S. Subgingival occurrence and antimicrobial susceptibility of enteric rods and pseudomonads from Brazilian periodontitis patients. Oral Microbiol Immunol 2001;16:306-10.

17. Botero JE, Arce RM, Escudero M, Betancourth M, Jaramillo, A, Contreras Al. Ocurrence of periodontopatic and superinfecting bacteria in chronic an aggressive periodontitis subject in a Colombia population. J Periodontol 2007;78:696-704

18. Lafaurie GI, Contreras A, Baron A, Botero J, MayorgaFayad I, Jaramillo A et al. Demographic, clinical, and microbial aspects of chronic and aggressive periodontitis in Colombia: a multicenter study. J Periodontol 2007;78:629-39.

19. Souto R, Colombo AP. Prevalence of Enterococcus faecalis in subgingival biofilm and saliva of subjects with chronic periodontal infection. Arch Oral Biol. 2008;53:155-60.

20. Albandar JM. Periodontal diseases in North America. Periodontol 2000 2002; 29: 31-69.

21. Sheiham A, Netuveli GS. Periodontal diseases in Europe. Periodontol 2000 2002;29:104-21. 
22. Armitage GC. Development of a classification system for periodontal diseases and conditions. Ann Periodontol 1999;4:1-6.

23. Slots J. Selective medium for isolation of Actinobacillus actinomycetemcomitans. J Clin Microbiol 1982;15:606-9.

24. Slots J. Rapid identification of important periodontal microorganisms by cultivation. Oral Microbiol Immunol 1986;1:48-55.

25. Koneman EW. Diagnóstico microbiológico. Texto y Atlas color. 5a ed. Madrid: Editorial Médica Panamericana; 1999.

26. Hamlet SM, Cullinan MP,Westerman B, Lindeman M, Bird PS, Palmer J et al. Distribution of Actinobacillus actinomycetemcomitans, Porphyromonas gingivalis and Prevotella intermedia in an Australian population. J Clin Periodontol 2001;28:1163-71.

27. Cortelli JR, Cortelli SC, Jordan S, Haraszthy VI, Zambon $\mathrm{JJ}$. Prevalence of periodontal pathogens in Brazilians with aggressive or chronic periodontitis. J Clin Periodontol 2005;32:860-6.

28. Ximenez-Fyvie LA, Almaguer-Flores A, Jacobo-Soto V, Lara-Cordoba M, Sanchez-Vargas LO, Alcantara-Maruri E. Description of the subgingival microbiota of periodontally untreated Mexican subjects: chronic periodontitis and periodontal health. J Periodontol 2006;77:460-71.

29. López NJ, Socransky SS, Da Silva I, Japlit MR, Haffajee AD. Subgingival microbiota of chilean patients with chronic periodontitis. J Periodontol 2004;75:717-25.

30. Riggio MP, Macfarlane TW, Mackenzie D, Lennon A, Smith AJ, Kinane D. Comparison of polymerase chain reaction and culture methods for detection of Actinobacillus actinomycetemcomitans and Porphyromonas gingivalis in subgingival plaque samples.J Periodontal Res 1996;31:496-501.

31. Lau L, Sanz M, Herrera D, Morillo JM, Martín C, Silva A. Quantitative real-time polymerase chain reaction ver- sus culture: a comparison between two methods for the detection and quantification of Actinobacillus actinomycetemcomitans, Porphyromonas gingivalis and Tannerella forsythensis in subgingival plaque samples. J Clin Periodontol 2004;31:1061-9.

32. Wu YM, Yan J, Chen LL, Gu ZY. Association between infection of different strains of Porphyromonas gingivalis and Actinobacillus actinomycetemcomitans in subgingival plaque and clinical parameters in chronic periodontitis. J Zhejiang Univ Sci B. 2007;8:121-31.

33. Takamatsu N, Yano K, He T, Umeda M, Ishikawa I. Effect of initial periodontal therapy on the frequency of detecting Bacteroides forsythus, Porphyromonas gingivalis, and Actinobacillus actinomycetemcomitans. J Periodontol 1999;70:574-80.

34. Ehmke B, Moter A, Beikler T, Milian E, Flemmig TF. Adjunctive antimicrobial therapy of periodontitis: longterm effects on disease progression and oral colonization. J Periodontol 2005;76:749-59.

35. Gonçalves MO, Coutinho-Filho WP, Pimenta FP, Pereira GA, Pereira JA, Mattos-Guaraldi AL et al. Periodontal disease as reservoir for multi-resistant and hydrolytic enterobacterial species. Lett Appl Microbiol 2007;44: 488-94.

36. Colombo AP, Teles RP, Torres MC, Souto R, Rosalém WJ, Mendes MC et al. Subgingival microbiota of Brazilian subjects with untreated chronic periodontitis. J Periodontol 2002;73:360-69.

37. Slots J, Feik D, Rams TE. Age and sex relationships of superinfecting microorganisms in periodontitis patients. Oral Microbiol Immunol 1990; 5: 305-8.

\section{CORRESPONDENCIA}

\author{
Carlos M. Ardila Medina \\ Calle $64 \mathrm{~N}^{\circ}$ 52-59 \\ Medellín, Colombia.
}

e-mail: martinardila@gmail.com 